\title{
ANCA Status or Clinical Phenotype — What Counts More?
}

\author{
Martin Windpessl ${ }^{1,2} \cdot$ Erica L. Bettac $^{3} \cdot$ Philipp Gauckler $^{4} \cdot$ Jae II Shin ${ }^{5} \cdot$ Duvuru Geetha $^{6} \cdot$ Andreas Kronbichler $^{4}$ (D)
}

Accepted: 23 March 2021 / Published online: 28 April 2021

(C) The Author(s) 2021

\begin{abstract}
Purpose of Review There is ongoing debate concerning the classification of antineutrophil cytoplasmic antibody (ANCA)associated vasculitis. That is, whether classification should be based on the serotype (proteinase 3 (PR3)- or myeloperoxidase (MPO)-ANCA) or on the clinical phenotype (granulomatosis with polyangiitis (GPA) or microscopic polyangiitis (MPA)). To add clarity, this review focused on integration of the most recent literature.

Recent Findings Large clinical trials have provided evidence that a serology-based risk assessment for relapses is more predictive than distinction based on the phenotype. Research conducted in the past decade indicated that a serology-based approach more closely resembles the genetic associations, the clinical presentation (i.e., lung involvement), biomarker biology, treatment response, and is also predicting comorbidities (such as cardiovascular death).

Summary Our review highlights that a serology-based approach could replace a phenotype-based approach to classify ANCAassociated vasculitides. In future, clinical trials and observational studies will presumably focus on this distinction and, as such, translate into a "personalized medicine."
\end{abstract}

Keywords Vasculitis $\cdot$ ANCA $\cdot$ Granulomatosis with polyangiitis $\cdot$ Microscopic polyangiitis $\cdot$ AAV

\section{Introduction}

Since the groundbreaking work of van de Woude et al. [1] in the 1980s, antineutrophil cytoplasmic antibodies (ANCA) have become indispensable in the diagnostic workup of vasculitides.

This article is part of the Topical Collection on Vasculitis

Andreas Kronbichler

andreas.kronbichler@i-med.ac.at

1 Department of Internal Medicine IV, Section of Nephrology, Klinikum Wels-Grieskirchen, Grieskirchnerstrasse 42, 4600 Wels, Austria

2 Medical Faculty, Johannes Kepler University Linz, Altenberger Strasse 69, 4040 Linz, Austria

3 Department of Psychology, Washington State University Vancouver, 14204 NE Salmon Creek Avenue, Vancouver, WA, USA

4 Department of Internal Medicine IV (Nephrology and Hypertension), Medical University Innsbruck, Anichstrasse 35, 6020 Innsbruck, Austria

5 Department of Pediatrics, Yonsei University College of Medicine, Seoul 03722, Republic of Korea

6 Division of Nephrology, Department of Medicine, Johns Hopkins University School of Medicine, Baltimore, MD, USA
They are useful for classification purposes and serve as markers of disease activity as well as predictors of relapses, all with due limitations [2]. Two major ANCA types exist: directed against proteinase 3 (PR3) and myeloperoxidase (MPO). ANCAassociated vasculitides (AAV) are a group of autoimmune disorders characterized by necrotizing inflammation of small- to medium-sized blood vessels, with a propensity for lung and kidney involvement. Granulomatosis with polyangiitis (GPA) and microscopic polyangiitis (MPA) are the two major clinical syndromes. PR3-ANCA is mainly associated with GPA and MPO-ANCA with MPA. Substantial overlap exists and discordant ANCA type (e.g., MPO-positive GPA) is sometimes observed in certain populations, particularly in Asia [3, 4].

The traditional nomenclature is predominantly based on the clinical phenotype. Despite considerable overlap, clear differences between PR3-AAV and MPO-AAV regarding epidemiologic and genetic background, as well as clinical course, have recently become apparent. There is growing recognition that the ANCA status (PR3 or MPO), rather than disease type (GPA or MPA), yields more valid clinical information. Current clinical trials already account for this by stratifying enrollment according to ANCA serotype [5••]. Indeed, the last decade has seen many developments in disease pathogenesis, biomarkers, and risk stratification. Thus, in the present review, 
we outline recent advancements in the field and propose how diseases summarized under the umbrella of AAV might be best classified. This review will not only help to clarify current underpinnings of AAV but will also inform future directions of research.

\section{AAV - Nomenclature and General Aspects}

The AAV share many similarities in mode of presentation, clinical course, and response to treatment. Accordingly, for a long time, GPA and MPA have been regarded as parts of a single disease spectrum and were frequently studied together in clinical trials $[5 \cdot, 6,7]$. To a lesser extent, ANCA have also been found in a proportion of patients with eosinophilic GPA (EGPA), with the majority of which exhibiting MPO-ANCA. Although EGPA is presently also included in the 2012 Chapel Hill Consensus Conference (CHCC2012) nomenclature of AAV [8], it is widely considered a separate entity and was not included in major AAV trials [9]. It is therefore not addressed in this review.

While the majority of patients with AAV are positive for one ANCA subtype, a few patients have both PR3- and MPOANCA. A study from Korea found 10 cases of dual positivity in a cohort of 85 patients with biopsy-confirmed AAV. Kidney dysfunction was more pronounced in subjects with dual positivity, and upper airway involvement occurred more frequently in this group as compared to MPO-ANCA positive patients [10]. ANCA are also present in the serum of one-third of patients with anti-GBM disease ("double-positive" serology) [11]. A study from four European centers found that in this subgroup of patients (in total 646 cases, 568 with singlepositive AAV, 41 with single-positive anti-GBM-disease, and 37 patients who were double positive), anti-MPO antibodies were more prevalent than anti-PR3 antibodies (70\% vs. $27 \%$ ) [12••]. Such individuals express a mixed clinical phenotype with disease severity at onset resembling pure anti-GBM disease and a propensity for relapse that is characteristic for AAV.

As proposed by the CHCC2012, vasculitides are classified according to the caliber of involved vessels. There is longstanding debate as to whether the clinical phenotype is an ideal way for classification. The disagreement among experts results from the fact that close similarities exist between GPA and MPA from a clinical point of view, and some contend categorization should follow ANCA serotype [13]. CHCC2012 thus recommended the addition of ANCA type as a prefix to the clinical phenotype (i.e., MPO-ANCA MPA, PR3-ANCA GPA, or ANCA-negative AAV) [8]. Even allowing some overlap, in single cases, it may be difficult to reconcile a positive ANCA serology with vessel size, as illustrated by a recent publication of the French Vasculitis Study Group (FVSG). In this nationwide case control study, Delaval et al. [14•] described 50 patients with AAV and temporal artery involvement, i.e., large vessel vasculitis (confirmed by biopsy in 42/50, where small-branch vasculitis and fibrinoid necrosis were distinguishing features). Despite an initial suspicion of giant cell arteritis, a diagnosis of AAV was ultimately maintained.

The prevalence of AAV is increasing, in part due to better awareness and more liberal ANCA testing, in part because of a higher survival of patients due to better therapies, but in part also due to a rise of the frequency of the disease. In spite of the bristling pace of evolution of treatment regimens, AAV still carry a poor prognosis, with a fatality rate of $28 \%$ at 5 years and survivors facing substantial morbidity resulting from the disease and its therapies [15]. The etiology of AAV is still unknown.

\section{ANCA Testing}

ANCA are autoantibodies (predominantly of the immunoglobulin $\mathrm{G}(\mathrm{IgG})$ isotope) directed against cytoplasmic antigens expressed in the primary granules of neutrophils and lysosomes of monocytes. ANCA are regarded as pathogenic, as convincingly shown in animal models for the MPO-ANCA subtype [16]; they can activate neutrophils and monocytes, thereby mediating vascular inflammation.

ANCA remain the most valuable diagnostic serological marker in vasculitis, but - as holds true for all biomarkers - they lack absolute specificity and sensitivity and must therefore be interpreted in the context of the full clinical picture. In other words, a negative ANCA test does not rule out a diagnosis of AAV when the scenario is suggestive, especially in patients with non-renal or locally limited disease [17]. Up to a third of patients with a clinical presentation compatible with AAV are ANCAnegative by routine testing [18]. On the other hand, ANCA positivity can also occur in the absence of clinically manifest vasculitis, for instance, in cases with infections, malignancy, or secondary to certain drugs. A particular association has been reported for levamisole-altered cocaine or the prescription of drugs used in the management of hyperthyroidism (propylthiouracil, methimazole, or carbimazole) [19-21]. In these situations, ANCA directed against MPO are more frequently found than antibodies against PR3 [22, 23].

Two methods exist for ANCA detection: (1) the traditional immunofluorescence test on fixed neutrophils (IF) and (2) enzyme-linked immunosorbent assays (ELISA). In AAV, IF typically exhibits two staining patterns, scored as cytoplasmic, with proteinase 3 (PR3) as the antigen, or as perinuclear, with myeloperoxidase (MPO) specificity. A two-step diagnostic approach (IF followed by ELISA) was the conventional laboratory method. A multicenter study from the European Vasculitis Society (EUVAS) [24] compared the results of two IF methods and 8 different immunoassays. Sera from 
251 incident AAV patients were compared with those of $>$ 900 patients in whom ANCA testing had been performed but vasculitis subsequently excluded. Strikingly, most new generation ELISA outperformed IF for ANCA determination. Given the excellent performance of modern immunoassays, an international expert consensus recommended initial testing with high-quality ELISA and posited that screening with IF could be abandoned in many cases [25]. A recent metaanalysis found a superior immunoassay sensitivity and confirms that screening for PR3- or MPO-ANCA improves the diagnostic workup of AAV [26], thus indicating that IF testing and a two-step approach are not needed.

\section{Novel Findings in Genetics and Pathomechanisms}

The pathogenesis of AAV is multifactorial and incompletely understood; however, a genetic component undoubtedly exists. There is compelling evidence that patients with MPOand PR3-ANCA serotype exhibit different genetic association [27]. Published in 2012, the first genome-wide association study (GWAS) has provided robust evidence that GPA and MPA are different diseases and that the ANCA serotype more strongly correlates with genetic associations compared to the disease entities, further fueling the concept of a serologybased classification [28].

Further, the role of complement in the pathogenesis of AAV has only recently received attention. In a study including 98 patients (45 MPO-ANCA positive, 53 PR3-ANCA positive), $\mathrm{Wu}$ et al. [29・•] studied activation of the alternative pathway in detail. Main differences in complement activation profile correlated predominantly with disease activity and barely with ANCA serotype. A recent study including a meta-analysis indicated that circulating complement activation products are elevated in both diseases, GPA and MPA, independent of clinical phenotype or ANCA serotype, indicating that complement activation is crucially involved in the pathogenesis of both diseases [30].

Monitoring the inflammatory status of the diseases using C-reactive protein (CRP) and erythrocyte sedimentation rate (ESR) is helpful. Both parameters tend to be higher among patients with PR3- as compared to patients with MPO-ANCA [31]. Using data from the RAVE trial, Berti et al. [32••] reported distinct cytokine profiles in patients with PR3- and MPO-ANCA positivity. Specifically, of the 186 cases included in the study, 124 exhibited PR3-ANCA, and the remainder MPO-ANCA. In this cohort of patients with active disease, immune mediators were more strongly associated with ANCA specificity than with clinical features, again underpinning the importance of ANCA in classifying these diseases.

\section{Differences in Demographic Factors and Manifestation}

ANCA status is also a major determinant of the clinical presentation. Although all age groups can be affected, PR3ANCA positive patients are on average 10 years younger than patients with MPO-ANCA; likewise, PR3-ANCA is significantly associated with male gender $[33,34]$. Ethnicity has a role in disease manifestation, as illustrated by the predominance of MPO-ANCA among patients with either GPA or MPA in Asia.

More than 20 years ago, Franssen et al. [13] reported a higher number of extrarenal organ involvement in PR3ANCA positive patients compared with their MPO-positive counterparts (mean number of affected organs at presentation 3.9 vs. 2.2, respectively) leading to a higher initial BVAS score in the former as compared to the latter. A large study involving 502 patients with AAV confirmed these findings [35]. These authors were also among the first to recommend that ANCA serotype should be used in conjunction with the terms MPA, GPA, and pauci-immune glomerulonephritis (GN), in categorizing patients within the AAV spectrum [35].

Ear, nose, and throat, as well as eye involvement, are more common in GPA and PR3-ANCA positive patients [31]. A recent cross-sectional study using data from the Diagnostic and Classification Criteria in Vasculitis (DCVAS) study underlined the importance of skin lesions, which occur in a third of patients with AAV at presentation. Cutaneous manifestations correlate with disease severity in patients with PR3ANCA but not in MPO-ANCA positive subjects. In the former group, petechiae/purpura were the most frequent findings (18.6\%), while the latter more frequently exhibited livedo reticularis and livedo racemosa. This may represent a useful distinguishing feature [36].

As the predominantly affected organ systems, specific differences of kidney and lung involvement will be discussed more extensively in the following sections.

\section{Kidney Involvement}

Renal vasculitis, i.e., pauci-immune rapidly progressive crescentic GN, is a common manifestation of AAV, affecting > $75 \%$ of patients in the early course of the disease [37, 38]. Seen from the other side, AAV-GN accounts for the majority (e.g., $80 \%$ in a contemporary Korean cohort [34]) of cases of crescentic GN. It carries an unfavorable prognosis, as it confers increased risks of end-stage kidney disease (ESKD) and mortality $[15,39,40]$. ANCA are nearly always present in AAV-GN, and a titer rise better predicts relapse in patients with than in patients without renal disease [2].

Overall, prevalence of kidney involvement appears to differ between PR3- and MPO-positive patients; 
moreover, important clinical differences exist between patients with these two serotypes. As found in recent literature, MPO-ANCA positive patients frequently have more advanced kidney disease at presentation and show a lesser likelihood of renal recovery following treatment. A recent publication from Weiner et al. [34] reported higher glomerular filtration rate at diagnosis in PR3-ANCA positive patients. Another study, conducted by the EUVAS group, analyzed 173 kidney biopsies and reported more pronounced glomerulosclerosis, interstitial fibrosis (IF), and tubular atrophy (TA) in individuals with MPO- than in those with PR3-ANCA. In contrast, the proportion of fibrinoid necrosis or crescents was similar in both groups [41, 42]. Therefore, in AAV, kidney biopsy is not only of value for diagnostic purposes but may also provide important prognostic information at baseline.

The histopathologic classification promoted in 2010 by a panel of kidney pathologists (Berden classification) distinguishes four categories of glomerular lesions: focal (> $50 \%$ normal glomeruli), crescentic (> 50\% cellular crescents), mixed (any other combination), and sclerotic (> $50 \%$ sclerotic glomeruli). Patients in the focal class tend to experience the best, while patients in the sclerotic class the worst renal outcomes [43]. It is important to note that neither data pertaining to ANCA serotype nor IF/TA or kidney function included in the score are available from this key publication, all of which have subsequently been shown to hold prognostic value too. In patients classified as sclerotic, entry GFR and TA seemed to correlate best with kidney function at 12 months and renal survival [44]. Long-term kidney survival, defined as outcome after 10 years, of the crescentic (86\%) and mixed class $(80 \%)$ is similar according to a recent publication. Kidney survival of the focal class was better, while the rate was lower in the sclerotic class [45 ${ }^{\bullet}$.

Several validation studies and a meta-analysis have been published since, predominantly confirming and expanding the findings described above [46]. Dividing patients by ANCA type, a large single-center study found the MPO-ANCA serotype was more frequent in the sclerotic class; furthermore, kidney survival was reduced in these subjects [47]. A subsequent publication confirmed these results in a larger, binational cohort of 136 patients [33]. Again, MPO-ANCA serotype was associated with more severe renal disease when compared with PR3-ANCA. Keeping with a more chronic or smoldering course, MPO-ANCA positivity was also associated with higher IF/TA scores, and more biopsies belonged to the sclerotic or mixed class [38]. At follow-up, ESKD and CKD stage 4 developed more frequently at 1 and 2 years in MPOANCA positive patients, compared to PR3-ANCA positive subjects. Further, a recent international study investigated predictors of renal outcomes in the sclerotic class, AAV-
GN. Of the 50 patients included, $41(82 \%)$ were positive for MPO-ANCA; kidney impairment was severe in this cohort with a median eGFR at entry of 14.5 (IQR 9-19) $\mathrm{ml} / \mathrm{min} / 1.73 \mathrm{~m}^{2}$ [44].

PR3-ANCA positive patients more often exhibit active inflammatory lesions yielding a better response to therapy $[18,41]$. Conversely, in MPO-AAV, worse kidney function implies smaller risk of relapse. This has implications for the optimal duration of maintenance therapy as these patients might not benefit from long-term immunosuppression. Recently, Brix et al. [48] proposed an "ANCA renal risk score" based on 3 clinicopathologic features, namely percentage of normal glomeruli, the amount of IF/TA, and baseline eGFR. Although the prevalence of ANCA serotype was balanced among study subjects, further analyses in larger cohorts are necessary to confirm that this score is applicable to both ANCA subtypes.

Renal limited vasculitis (RLV), i.e., isolated pauci-immune crescentic GN, is a distinct subset of AAV, in which MPOANCA positivity is found in $80 \%, 10 \%$ are PR3-ANCA positive, with the remainder seronegative [49]. Interestingly, a recent study using mass spectrometry found a more pronounced complement deposition in kidney biopsies of patients with ANCA-negative GN in comparison to PR3-ANCA or MPO-ANCA positive patients with GN. The authors hypothesize that ANCA-negative GN may be triggered or promoted by a defect in the alternative complement pathway, either genetically or acquired [50]. These findings await replication in prospective studies.

A recent study investigated cases with "slowly progressive" renal vasculitis (defined by a $<50 \%$ decrease of eGFR over a 6-month period prior diagnosis). These patients were frequently MPO-ANCA positive (37/39) and involvement of other organs was absent in most patients. Most kidney biopsies were either classified as sclerotic or mixed class. Immunosuppression led to an improvement of eGFR of $>$ $25 \%$ from diagnosis in $59 \%$ of patients [51].

In summary, kidney response to therapy appears to be better in PR3-ANCA positive patients and translates into a more favorable long-term prognosis. The renal course of MPO-AAV is characterized by a more chronic process, with global sclerosis and advanced fibrosis on histology bearing witness of a gradual accrual of damage. Therefore, the component of chronicity, rather than the degree of disease activity, provides better information regarding renal outcome [52].

A kidney biopsy exhibits prognostic value, while in cases with clinical presentations that are compatible with AAV, a positivity of either PR3- or MPO-ANCA and a low suspicion for secondary vasculitis forms, treatment can be initiated without a biopsy-confirmed diagnosis [53]. This is of particular interest in scenarios when centers aim to avoid biopsy (i.e., morbid obesity or presence of a single kidney) (Table 1). 
Table 1 Characteristics of kidney involvement in ANCA-associated vasculitis

\begin{tabular}{|c|c|c|}
\hline Characteristics of kidney involvement in AAV & MPO-ANCA & PR3-ANCA \\
\hline Baseline eGFR (at diagnosis) & Lower & Higher \\
\hline Histopathology & $\begin{array}{l}\text { Advanced damage; higher IF/TA scores; more } \\
\text { frequently "sclerotic" class (according to } \\
\text { the Berden classification) }\end{array}$ & $\begin{array}{l}\text { Active inflammatory lesions; more } \\
\text { frequently "focal" class } \\
\text { (according to the Berden classification) }\end{array}$ \\
\hline Renal limited vasculitis (proportion of patients) & $80 \%$ & $10 \%$ \\
\hline Treatment response (kidney function recovery) & Worse & Better \\
\hline Long-term prognosis & Worse, chronic process & Better \\
\hline Relapse risk & Lower & Higher \\
\hline
\end{tabular}

Abbreviations: $A A V$, ANCA-associated vasculitis; $M P O$, myeloperoxidase; $P R 3$, proteinase 3 ; $e G F R$, estimated glomerular filtration rate; $I F / T A$, interstitial fibrosis/tubular atrophy

\section{Pulmonary Involvement}

Lung involvement appears prominently among the various manifestations of AAV. At the most severe end of the disease spectrum, diffuse alveolar hemorrhage (DAH), as a consequence of capillaritis, carries a high mortality. Notably, the likelihood of DAH did not segregate according to ANCA specificity in a single-center cohort study of 73 patients [54]. Less dramatic pulmonary pathology ranges from segmental bronchial wall thickening, bronchiectasis, and single parenchymal nodules to multiple large pulmonary granulomas (primarily in patients with GPA) [55•].

An association of AAV with interstitial lung disease (ILD) was initially noted in 1990, and this observation was subsequently bolstered by various case series from Japan and other countries [56, 57]. A single-center study involving 53 patients with ILD who had ANCA measured found 19 ANCA positive patients, 17 of them exhibiting the MPO-ANCA subtype [58]. In a multicenter retrospective study, Comarmond et al. [59] described 49 patients with AAV and ILD. Male predominance was noted (30 men [61\%], and 28 subjects exhibited kidney involvement $[57 \%])$. Again, ANCA specificity was mostly MPO (43 [88\%]), with only 3 patients showing PR3-ANCA. Schirmer and colleagues [60] identified 144 patients with MPA from their Bad Bramstedt cohort, of which $12 \%$ demonstrated ILD at disease onset and 15\% during long-term follow-up. It has been repeatedly stressed that the evidence of ILD often predates the manifestation of systemic vasculitis. Specifically, patients with seemingly "idiopathic" pulmonary fibrosis may present with rapidly progressive GN years after the initial diagnosis of lung disease [61]. Seroconversion, i.e., the development of ANCA in previously autoantibody negative patients with ILD, is well recognized. In a cohort of 61 patients with idiopathic pulmonary fibrosis, 6 individuals became MPO-ANCA positive during follow-up, with a median duration of 23 months (range, 0 to 71 months); 2 patients developed crescentic GN and subsequently received a diagnosis of MPA [62].
Usual interstitial pneumonia (UIP) is the predominant radiologic pattern of MPA-ILD: the FVSG reported 62 patients with AAV-ILD (89\% MPO-ANCA subtype). ILD was defined as UIP $(63 \%)$ or non-specific interstitial pneumonia (NSIP) according to radiologic pattern [63]. In this cohort, UIP conferred a poor prognosis.

Individuals with MPA-ILD are usually older than patients with MPA without ILD and pure "vasculitic" presentation, i.e., acute onset of constitutional symptoms. Extant literature, albeit limited, consistently indicates a slight male preponderance.

Subclinical alveolar hemorrhage has been implicated as the mechanism leading to pulmonary fibrosis. In addition, MPOANCA per se may play a role in the pathogenesis of pulmonary fibrosis [64]. According to another hypothesis, the chronic inflammatory process of pulmonary fibrosis may result in neutrophil destruction and subsequent development of ANCA. Tobacco exposure appears to be an additional trigger, as a high prevalence of AAV is consistently found in former or current smokers, especially among MPO-ANCA positive individuals. In contrast, no such association was found in patients with PR3-ANCA positive AAV [65••].

There is no specific treatment for AAV-associated ILD, and available literature is conflicting concerning the possible benefit of immunosuppressive therapy [64]. Nintedanib, a tyrosine kinase inhibitor, is an interesting agent in this indication given its efficacy in scleroderma lung disease [66].

PR3-ANCA positive patients with lung involvement exhibit a substantially increased risk of relapse when compared to subjects with MPO-ANCA; it is speculated that this risk can be partly reduced by trimethoprim/sulfamethoxazole, presumably by preventing respiratory tract infections [67].

To conclude, pulmonary fibrosis can be associated with MPO-AAV, and it appears to be a distinct manifestation causing increased disability and poor prognosis. Greater awareness of this association and more liberal testing for ANCA in ILD may result in an earlier recognition and, ultimately, improved outcomes. Close collaboration between pulmologists, rheumatologists, and nephrologists is clearly necessary. 


\section{Treatment Response and Risk of Relapse}

The treatment armamentarium has increased during the past years, and a particular focus was to reduce the risk of relapse once remission has been obtained by induction therapy. A study by Mahr et al. summarized findings from different clinical trials conducted by EUVAS and FVSG and indicated a higher risk of disease relapse in patients with PR3-ANCA than in other AAV patients [68]. Continuous improvements in treatment strategies have transformed AAV from an almost inevitably fatal disease to an entity with a relapsing and remitting course; concomitantly, treatment-related toxicity has emerged as a major concern. Following the publication of RITUXVAS and RAVE, two key trials paving the way for authorization of rituximab for the treatment of AAV [6, 7], significant differences in the response to treatment in AAV have become apparent. While the RAVE trial found non-inferiority of rituximab (RTX) in the induction of remission, compared to a cyclophosphamide (CYC)-based regime, the presence of PR3-ANCA (as compared to MPO-ANCA) portended a better response to RTX than to CYC [6]. This came at the cost of an increased relapse risk, necessitating prolonged maintenance therapy. Major guidelines now recommend a longer course of maintenance therapy in patients with PR3-ANCA and advocate that RTX should be given preference over CYC in induction of such cases $[69,70]$. In MAINRITSAN3, a trial assessing the benefit of prolonged maintenance therapy with RTX, relapses were common in patients with PR3ANCA vasculitis in the placebo group, underscoring the importance of long-term treatment with rituximab in these patients to prevent disease recurrences [71•]. In addition, there may be differences in response to mycophenolate mofetil (MMF) in MPO-ANCA compared to PR3ANCA patients. The MYCYC trial (comparing MMF versus $\mathrm{CYC}$ for induction of remission in AAV) demonstrated non-inferiority of MMF in comparison to CYC. The study included 140 patients, with MPO-ANCA positive subjects evenly split between the two treatment arms (27/70 MMF group, 26/70 CYC group). A post-hoc analysis found no difference between MMF and CYC in the relapse rate of MPO-positive cases, while relapse risk in the PR3-ANCA vasculitis patients tended to be higher in the MMF arm [72].

Studies assessing novel steroid-sparing strategies are underway with avacopan, an antagonist of human C5aR/CD88 being one of the most promising drugs. It has shown promising results in a phase 2 trial (CLEAR), showing comparable efficacy by replacing high dose glucocorticoids [73••]. Numerically, the response rate was greater in subjects with MPO-ANCA vasculitis. The results of a large phase 3 trial (ADVOCATE) are awaited. Lastly, the PEXIVAS trial [5••] did not report any effect of ANCA type on the response to plasma exchange or on the two different corticosteroid arms.

Overall, predictors of relapse such as ANCA type have influenced treatment algorithms and should allow for a more individualized long-term therapeutic approach.

\section{Cardiovascular Disease and Venous Thromboembolism}

With inflammation of blood vessels as their histological hallmark feature, it is not surprising that AAV confer an increased risk of cardiovascular (CV) morbidity and mortality [74]. The rate of CV events ( $20 \%$ within 40 months following diagnosis) even exceeds the number observed in patients with CKD, a cohort prone to CV morbidity [75]. A recent publication, including 484 patients with a new diagnosis of AAV between 2002 and 2017, found a greater risk of death due to CV disease in MPO-ANCA+ patients compared with PR3-ANCA+ patients. The association persisted after adjustment for baseline kidney function, age, and sex [76••]. This paper corroborated an earlier publication from the EUVAS, as well as the FVSG, which highlighted (1) the increased risk of death of patients with CV involvement and (2) that the risk of death is higher in patients without PR3-ANCA [68]. A recent post-hoc analysis of the RAVE trial investigated changes in the lipid profile of patients from baseline to month 6 . In patients with newly diagnosed AAV, there was a significant increment in lowdensity lipoprotein (LDL)-cholesterol following remission induction, and these changes were most evident in patients with PR3-ANCA vasculitis [77]. This finding implies that the individual $\mathrm{CV}$ disease risk should be assessed once remission is achieved. In addition, a German cross-sectional multicenter study focused on the management of $\mathrm{CV}$ risk factors in patients with AAV. By using guidelines issued by several authorities to treat hyperlipidemia (KDIGO and ESC), only a minority $(<25 \%)$ of the 53 patients reached their individual target LDL-cholesterol [78]. More recently, target LDLcholesterol levels and blood pressure for patients at high risk for $\mathrm{CV}$ events have been lowered. More studies in the field of $\mathrm{AAV}$ are needed to provide evidence that a more stringent control of $\mathrm{CV}$ disease risk factors can prevent events.

A hypercoagulable state of patients with AAV was reported during phases of active disease and remission. The frequency of venous thromboembolic events (VTE) is up to $17.3 \%$ (Table 2), as reported in a recent study focusing on patients with ANCA-associated GN. The median time between diagnosis of vasculitis and VTE was 3 months. In this study, the absence of statin therapy was associated with an increased risk of VTE, a finding which needs to be confirmed by additional research [87]. Post-hoc analysis of the RAVE trial indicated that 16 of 197 patients had at least one event and that most VTE occurred early after enrolment (with an overall average 


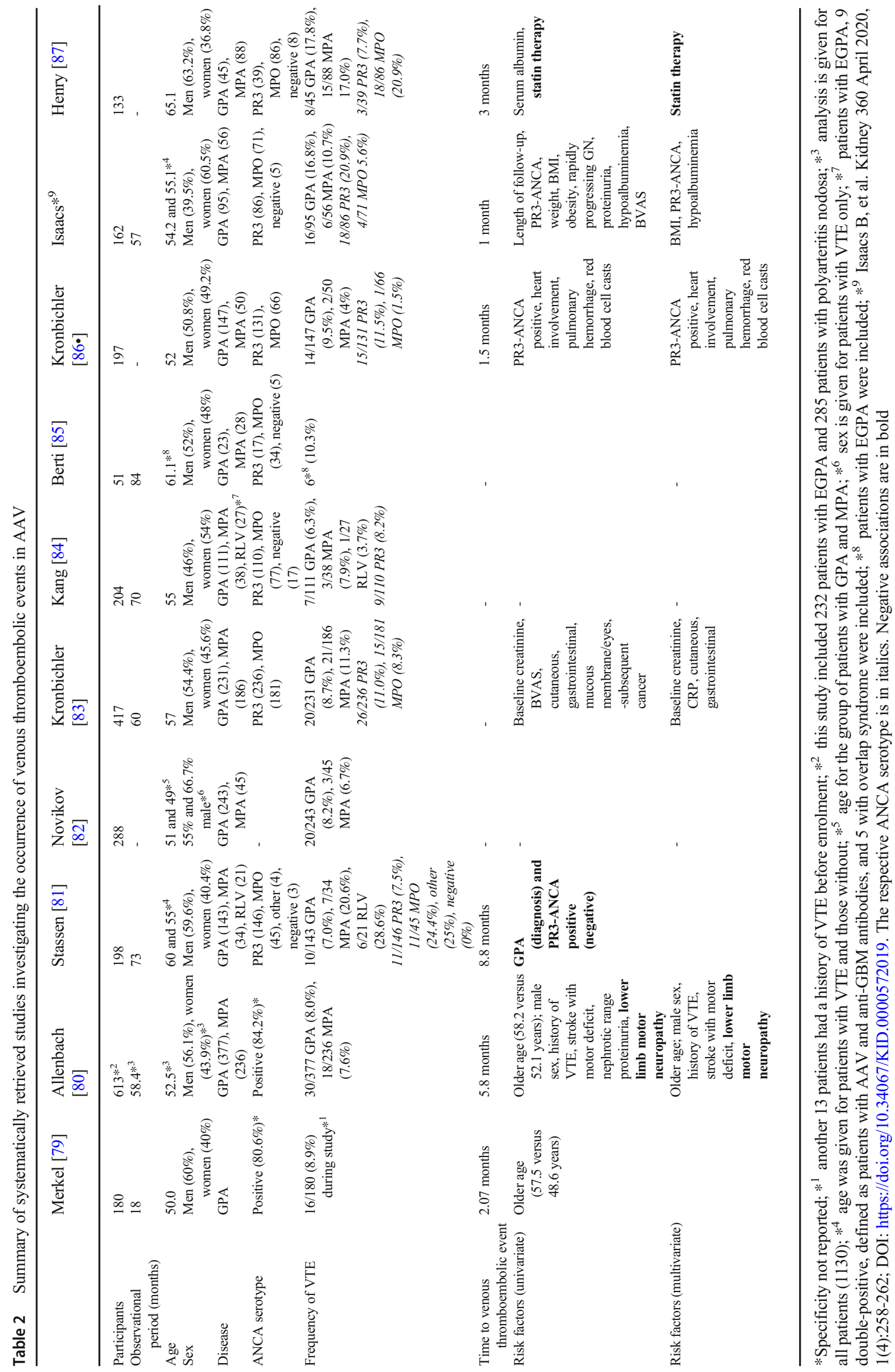


time to event of 1.5 months). A strong association of VTE with PR3-ANCA positivity, pulmonary hemorrhage, and urinary red blood cell casts was reported [86 ${ }^{\circ}$. In contrast, Stassen et al. found an association of VTE with MPOANCA positivity and a diagnosis of MPA. No adjustment for biases such as older age of the participants was made for this finding [81]. A potential mechanism which predisposes patients with PR3-ANCA vasculitis to VTE was published by Bautz et al., showing that patients with PR3-ANCA positivity have higher anti-plasminogen antibody levels compared to MPO-positive patients. Moreover, five of nine patients with VTE in the PR3-ANCA group exhibited anti-plasminogen antibodies; in contrast, these were absent in patients with MPO-ANCA vasculitis and thrombosis [88]. Patients who achieved remission had impaired levels of endogenous thrombin potential, factor VIII, and tissue factor pathway inhibitor, which indicate the hypercoagulable state persists during remission [89]. While overall control of CV risk factors by lipid-lowering agents such as statins might be considered, we are not recommending initiation of statins to prevent VTE. Evidence from the general population stems from observational studies and should form the basis for studying their effects in properly designed interventional trials [90].

In summary, as treatment approaches allow for control of the disease in most patients, a particular focus is on comorbidities and complications of the diseases. CV mortality is high and a better control of risk factors seems warranted. A specific approach to reduce the burden of thromboembolic events needs to be developed, and clinical trials should be initiated to reduce the occurrence of VTE in AAV patients.

\section{Summary}

The past years have witnessed impressive advances in the understanding of AAV, and from this, considerable improvements in patient care have followed. In parallel, a shift in classification has occurred, moving away from phenotype (GPA or MPA) and focusing instead on ANCA serotype. There is now largely consensus that PR3-AAV and MPOAAV represent separate entities in many practical regards and differ genetically. Currently, there are no validated diagnostic criteria for AAV, likewise, an ideal classification of this entity remains elusive and relevant questions remain unsolved. For instance, the pattern of organ involvement does not always follow ANCA serology clearly. Moreover, up to a third of patients with features consistent with AAV are ANCA-negative by routine tests, and how to best treat and follow-up such patients currently remains unclear. The ongoing DCVAS study (NCT01066208) [91], a multinational, observational study to develop diagnostic and update classification criteria in vasculitis, is expected to settle some of these issues.
As is true for all diseases whose spectrum is broad, caution is required in all efforts to ascribe patients to simple disease subsets. New insights into pathophysiology, such as the role of complement in AAV, might impact future categorization. Such influence would emulate the discovery of ANCA almost 40 years ago.

The general classification of the vasculitides, and of the AAV in particular, will continue to evolve. Better insight into individual patient risk factors for disease, response to therapy, and risk of adverse events may result from this. Currently, widespread uptake of an ANCA-based classification of the AAV could rapidly usher an era of personalized medicine in this field. Such patient-tailored therapy could enable a more individualized approach with better treatment response and less therapy-related side effects ultimately resulting in improved patient-related outcomes.

Funding Open access funding provided by University of Innsbruck and Medical University of Innsbruck.

\section{Declarations}

Conflict of Interest Dr. Geetha reports personal fees (consultancy) from ChemoCentryx and Aurinia, outside the submitted work. Dr. Kronbichler reports personal fees from Novartis, from Vifor Pharma, and from TerumoBCT (speaker honorarium), outside the submitted work. Martin Windpessl, Erica L. Bettac, Philipp Gauckler, and Jae Il Shin declare that they have no conflict of interest.

Human and Animal Rights and Informed Consent This article does not contain any studies with human or animal subjects performed by any of the authors.

Open Access This article is licensed under a Creative Commons Attribution 4.0 International License, which permits use, sharing, adaptation, distribution and reproduction in any medium or format, as long as you give appropriate credit to the original author(s) and the source, provide a link to the Creative Commons licence, and indicate if changes were made. The images or other third party material in this article are included in the article's Creative Commons licence, unless indicated otherwise in a credit line to the material. If material is not included in the article's Creative Commons licence and your intended use is not permitted by statutory regulation or exceeds the permitted use, you will need to obtain permission directly from the copyright holder. To view a copy of this licence, visit http://creativecommons.org/licenses/by/4.0/.

\section{References}

Papers of particular interest, published recently, have been highlighted as:

- Of importance

• Of major importance

1. van der Woude FJ, Rasmussen N, Lobatto S, Wiik A, Permin H, van Es LA, et al. Autoantibodies against neutrophils and 
monocytes: tool for diagnosis and marker of disease activity in Wegener's granulomatosis. Lancet. 1985;1(8426):425-9.

2. Kemna MJ, Damoiseaux J, Austen J, Winkens B, Peters J, van Paassen P, et al. ANCA as a predictor of relapse: useful in patients with renal involvement but not in patients with nonrenal disease. $\mathrm{J}$ Am Soc Nephrol. 2015;26(3):537-42.

3. Schirmer JH, Wright MN, Herrmann K, Laudien M, Nölle B, Reinhold-Keller E, et al. Myeloperoxidase-antineutrophil cytoplasmic antibody (ANCA)-positive granulomatosis with polyangiitis (Wegener's) is a clinically distinct subset of ANCA-associated vasculitis: a retrospective analysis of 315 patients from a German vasculitis referral center. Arthritis Rheumatol. 2016;68(12):2953-63.

4. Chen M, Yu F, Zhang Y, Zou WZ, Zhao MH, Wang HY. Characteristics of Chinese patients with Wegener's granulomatosis with anti-myeloperoxidase autoantibodies. Kidney Int. 2005;68(5): 2225-9.

5.• Walsh M, Merkel PA, Peh CA, Szpirt WM, Puéchal X, Fujimoto $\mathrm{S}$, et al. Plasma exchange and glucocorticoids in severe ANCAassociated vasculitis. N Engl J Med. 2020;382(7):622-31. Plasma exchange did not decrease the composite of end-stage kidney disease or death in severe ANCA-associated vasculitis, but a reduced-dose regimen of glucocorticoids was non-inferior compared to the standard-dosed regimen, with a reduced occurrence of severe infections at 1 year.

6. Stone JH, Merkel PA, Spiera R, Seo P, Langford CA, Hoffman GS, et al. Rituximab versus cyclophosphamide for ANCA-associated vasculitis. N Engl J Med. 2010;363(3):221-32.

7. Jones RB, Tervaert JW, Hauser T, Luqmani R, Morgan MD, Peh $\mathrm{CA}$, et al. Rituximab versus cyclophosphamide in ANCAassociated renal vasculitis. N Engl J Med. 2010;363(3):211-20.

8. Jennette JC, Falk RJ, Bacon PA, Basu N, Cid MC, Ferrario F, et al. 2012 revised International Chapel Hill Consensus Conference nomenclature of vasculitides. Arthritis Rheum. 2013;65(1):1-11.

9. Groh M, Pagnoux C, Baldini C, Bel E, Bottero P, Cottin V, et al. Eosinophilic granulomatosis with polyangiitis (Churg-Strauss) (EGPA) Consensus Task Force recommendations for evaluation and management. Eur J Intern Med. 2015;26(7):545-53.

10. Kim SM, Choi SY, Kim SY, Kim J. Clinical characteristics of patients with vasculitis positive for anti-neutrophil cytoplasmic antibody targeting both proteinase 3 and myeloperoxidase: a retrospective study. Rheumatol Int. 2019;39(11):1919-26.

11. Rutgers A, Slot M, van Paassen $P$, van Breda Vriesman $P$, Heeringa P, Tervaert JW. Coexistence of anti-glomerular basement membrane antibodies and myeloperoxidase-ANCAs in crescentic glomerulonephritis. Am J Kidney Dis. 2005;46(2):253-62.

12. McAdoo SP, Tanna A, Hrušková Z, Holm L, Weiner M, Arulkumaran N, et al. Patients double-seropositive for ANCA and anti-GBM antibodies have varied renal survival, frequency of relapse, and outcomes compared to single-seropositive patients. Kidney Int. 2017;92(3):693-702. Description of double-positive patients (ANCA-associated vasculitis + anti-GBM disease) as a hybrid disease, with features of both diseases (older age distribution, diagnostic delay, severe kidney disease, and lung hemorrhage at presentation).

13. Franssen CF, Stegeman CA, Kallenberg CG, Gans RO, De Jong PE, Hoorntje SJ, et al. Antiproteinase 3- and antimyeloperoxidaseassociated vasculitis. Kidney Int. 2000;57(6):2195-206.

14. Delaval L, Samson M, Schein F, Agard C, Tréfond L, Deroux A, et al. Temporal arteritis revealing antineutrophil cytoplasmic antibody-associated vasculitides: case-control study of 50 cases. Arthritis Rheumatol. 2021;73(2):286-94. https://doi.org/10.1002/ art.41527. Cases with temporal arteritis and underlying ANCA-associated vasculitis are described retrospectively, with a median diagnostic delay of 15 months in $60 \%$ of patients.

15. Rhee RL, Hogan SL, Poulton CJ, McGregor JA, Landis JR, Falk $\mathrm{RJ}$, et al. Trends in long-term outcomes among patients with antineutrophil cytoplasmic antibody-associated vasculitis with renal disease. Arthritis Rheumatol. 2016;68(7):1711-20.

16. Shochet L, Holdsworth S, Kitching AR. Animal models of ANCA associated vasculitis. Front Immunol. 2020;11:525.

17. Finkielman JD, Lee AS, Hummel AM, Viss MA, Jacob GL, Homburger HA, et al. ANCA are detectable in nearly all patients with active severe Wegener's granulomatosis. Am J Med. 2007;120(7):643-e9-14.

18. Cornec D, Cornec-Le Gall E, Fervenza FC, Specks U. ANCAassociated vasculitis - clinical utility of using ANCA specificity to classify patients. Nat Rev Rheumatol. 2016;12(10):570-9.

19. Muñoz-Vahos CH, Herrera-Uribe S, Arbeláez-Cortés Á, JaramilloArroyave D, González-Naranjo LA, Vásquez-Duque G, et al. Clinical profile of levamisole-adulterated cocaine-induced vasculitis/vasculopathy: a 30-case series. J Clin Rheumatol. 2019;25(3): e16-26.

20. Choi HK, Merkel PA, Walker AM, Niles JL. Drug-associated antineutrophil cytoplasmic antibody-positive vasculitis: prevalence among patients with high titers of antimyeloperoxidase antibodies. Arthritis Rheum. 2000;43(2):405-13.

21. Slot MC, Links TP, Stegeman CA, Tervaert JW. Occurrence of antineutrophil cytoplasmic antibodies and associated vasculitis in patients with hyperthyroidism treated with antithyroid drugs: a long-term followup study. Arthritis Rheum. 2005;53(1):108-13.

22. Moiseev S, Cohen Tervaert JW, Arimura Y, Bogdanos DP, Csernok E, Damoiseaux J, et al. 2020 international consensus on ANCA testing beyond systemic vasculitis. Autoimmun Rev. 2020;19(9):102618.

23. McAdoo SP, Hall A, Levy J, Salama AD, Pusey CD. Proteinase-3 antineutrophil cytoplasm antibody positivity in patients without primary systemic vasculitis. J Clin Rheumatol. 2012;18(7):336-40.

24. Damoiseaux J, Csernok E, Rasmussen N, Moosig F, van Paassen P, Baslund B, et al. Detection of antineutrophil cytoplasmic antibodies (ANCAs): a multicentre European Vasculitis Study Group (EUVAS) evaluation of the value of indirect immunofluorescence (IIF) versus antigen-specific immunoassays. Ann Rheum Dis. 2017;76(4):647-53

25. Bossuyt X, Cohen Tervaert JW, Arimura Y, Blockmans D, FloresSuárez LF, Guillevin L, et al. Position paper: revised 2017 international consensus on testing of ANCAs in granulomatosis with polyangiitis and microscopic polyangiitis. Nat Rev Rheumatol. 2017;13(11):683-92.

26. Guchelaar NAD, Waling MM, Adhin AA, van Daele PLA, Schreurs MWJ, Rombach SM. The value of anti-neutrophil cytoplasmic antibodies (ANCA) testing for the diagnosis of ANCAassociated vasculitis, a systematic review and meta-analysis. Autoimmun Rev. 2021;20(1):102716.

27. Sharma RK, Lövström B, Gunnarsson I, Malmström V. Proteinase 3 autoreactivity in anti-neutrophil cytoplasmic antibody-associated vasculitis-immunological versus clinical features. Scand J Immunol. 2020;92(5):e12958.

28. Lyons PA, Rayner TF, Trivedi S, Holle JU, Watts RA, Jayne DR, et al. Genetically distinct subsets within ANCA-associated vasculitis. N Engl J Med. 2012;367(3):214-23.

29.• Wu EY, McInnis EA, Boyer-Suavet S, Mendoza CE, Aybar LT, Kennedy KB, et al. Measuring circulating complement activation products in myeloperoxidase- and proteinase 3-antineutrophil cytoplasmic antibody-associated vasculitis. Arthritis Rheumatol. 2019;71(11):1894-903. Circulating complement activation products were measured in 98 patients, with higher levels of C3a, C5a, and sC5b-9 in active cases compared to controls. Some complement products remained elevated during remission phase.

30. Moiseev S, Lee JM, Zykova A, Bulanov N, Novikov P, Gitel E, et al. The alternative complement pathway in ANCA-associated 
vasculitis: further evidence and a meta-analysis. Clin Exp Immunol. 2020;202:394-402.

31. Mohammad AJ, Segelmark M. A population-based study showing better renal prognosis for proteinase 3 antineutrophil cytoplasmic antibody (ANCA)-associated nephritis versus myeloperoxidase ANCA-associated nephritis. J Rheumatol. 2014;41(7):1366-73.

32.• Berti A, Warner R, Johnson K, Cornec D, Schroeder D, Kabat B, et al. Brief report: circulating cytokine profiles and antineutrophil cytoplasmic antibody specificity in patients with antineutrophil cytoplasmic antibody-associated vasculitis. Arthritis Rheumatol. 2018;70(7):1114-21. A panel of 29 cytokines was tested in $\mathbf{1 8 6}$ patients recruited to the RAVE trial. Analysis found that the difference of cytokine expression was in most cases larger between PR3-ANCA vasculitis and MPO-ANCA vasculitis as compared to a phenotype (GPA and MPA)-based distinction.

33. Quintana LF, Peréz NS, De Sousa E, Rodas LM, Griffiths MH, Solé $\mathrm{M}$, et al. ANCA serotype and histopathological classification for the prediction of renal outcome in ANCA-associated glomerulonephritis. Nephrol Dial Transplant. 2014;29(9):1764-9.

34. Weiner M, Bjørneklett R, Hrušková Z, Mackinnon B, Poulton CJ, Sindelar L, et al. Proteinase-3 and myeloperoxidase serotype in relation to demographic factors and geographic distribution in anti-neutrophil cytoplasmic antibody-associated glomerulonephritis. Nephrol Dial Transplant. 2019;34(2):301-8.

35. Lionaki S, Blyth ER, Hogan SL, Hu Y, Senior BA, Jennette CE, et al. Classification of antineutrophil cytoplasmic autoantibody vasculitides: the role of antineutrophil cytoplasmic autoantibody specificity for myeloperoxidase or proteinase 3 in disease recognition and prognosis. Arthritis Rheum. 2012;64(10):3452-62.

36. Micheletti RG, Chiesa Fuxench Z, Craven A, Watts RA, Luqmani RA, Merkel PA, et al. Cutaneous manifestations of antineutrophil cytoplasmic antibody-associated vasculitis. Arthritis Rheumatol. 2020;72(10):1741-7. https://doi.org/10.1002/art.41310.

37. Booth AD, Almond MK, Burns A, Ellis P, Gaskin G, Neild GH, et al. Outcome of ANCA-associated renal vasculitis: a 5-year retrospective study. Am J Kidney Dis. 2003;41(4):776-84.

38. Franssen CF, Stegeman CA, Oost-Kort WW, Kallenberg CG, Limburg PC, Tiebosch A, et al. Determinants of renal outcome in anti-myeloperoxidase-associated necrotizing crescentic glomerulonephritis. J Am Soc Nephrol. 1998;9(10):1915-23.

39. Berti A, Cornec-Le Gall E, Cornec D, Casal Moura M, Matteson EL, Crowson CS, et al. Incidence, prevalence, mortality and chronic renal damage of anti-neutrophil cytoplasmic antibody-associated glomerulonephritis in a 20-year population-based cohort. Nephrol Dial Transplant. 2019;34(9):1508-17.

40. Flossmann O, Berden A, de Groot K, Hagen C, Harper L, Heijl C, et al. Long-term patient survival in ANCA-associated vasculitis. Ann Rheum Dis. 2011;70(3):488-94.

41. Hauer HA, Bajema IM, Van Houwelingen HC, Ferrario F, Noël LH, Waldherr R, et al. Determinants of outcome in ANCAassociated glomerulonephritis: a prospective clinicohistopathological analysis of 96 patients. Kidney Int. 2002;62(5): 1732-42.

42. Vizjak A, Rott T, Koselj-Kajtna M, Rozman B, Kaplan-Pavlovcic S, Ferluga D. Histologic and immunohistologic study and clinical presentation of ANCA-associated glomerulonephritis with correlation to ANCA antigen specificity. Am J Kidney Dis. 2003;41(3): 539-49.

43. Berden AE, Ferrario F, Hagen EC, Jayne DR, Jennette JC, Joh K, et al. Histopathologic classification of ANCA-associated glomerulonephritis. J Am Soc Nephrol. 2010;21(10):1628-36.

44. Menez S, Hruskova Z, Scott J, Cormican S, Chen M, Salama AD, et al. Predictors of renal outcomes in sclerotic class anti-neutrophil cytoplasmic antibody glomerulonephritis. Am J Nephrol. 2018;48(6):465-71.
45. van Daalen EE, Wester Trejo MAC, Göçeroğlu A, Ferrario F, Joh K, Noël LH, et al. Developments in the histopathological classification of ANCA-associated glomerulonephritis. Clin J Am Soc Nephrol. 2020;15(8):1103-11. https://doi.org/10.2215/CJN.14561119. Longterm kidney survival (8 years of follow-up) is different among the four histopathological classes (focal, crescentic, mixed, and sclerotic). Application of the ANCA kidney risk score indicated that those belonging to the low or medium risk groups have excellent 3-year kidney survival (100 and 96\%)

46. Chen YX, Xu J, Pan XX, Shen PY, Li X, Ren H, et al. Histopathological classification and renal outcome in patients with antineutrophil cytoplasmic antibodies-associated renal vasculitis: a study of 186 patients and metaanalysis. J Rheumatol. 2017:44(3): 304-13.

47. Tanna A, Guarino L, Tam FW, Rodriquez-Cubillo B, Levy JB, Cairns TD, et al. Long-term outcome of anti-neutrophil cytoplasm antibody-associated glomerulonephritis: evaluation of the international histological classification and other prognostic factors. Nephrol Dial Transplant. 2015;30(7):1185-92.

48. Brix SR, Noriega M, Tennstedt P, Vettorazzi E, Busch M, Nitschke $\mathrm{M}$, et al. Development and validation of a renal risk score in ANCA-associated glomerulonephritis. Kidney Int. 2018;94(6): $1177-88$.

49. Novick TK, Chen M, Scott J, Cortazar FB, Ayoub I, Little MA, et al. Patient outcomes in renal-limited antineutrophil cytoplasmic antibody vasculitis with inactive histology. Kidney Int Rep. 2018;3(3):671-6.

50. Sethi S, Zand L, De Vriese AS, Specks U, Vrana JA, Kanwar S, et al. Complement activation in pauci-immune necrotizing and crescentic glomerulonephritis: results of a proteomic analysis. Nephrol Dial Transplant. 2017;32(suppl_1):i139-i45.

51. Trivioli G, Gopaluni S, Urban ML, Gianfreda D, Cassia MA, Vercelloni PG, et al. Slowly progressive anti-neutrophil cytoplasmic antibody-associated renal vasculitis: clinico-pathological characterization and outcome. Clin Kidney J. 2020;14(1):332-40. https://doi.org/10.1093/ckj/sfaa139.

52. Smith JR, Methven S, Stevens KI, MacKinnon B, Kidder D. Renal risk score performance in a cohort of Scottish patients with ANCAassociated glomerulonephritis. Kidney Int. 2019;96(4):1037.

53. Jayne D. Vasculitis-when can biopsy be avoided? Nephrol Dial Transplant. 2017;32(9):1454-6.

54. Cartin-Ceba R, Diaz-Caballero L, Al-Qadi MO, Tryfon S, Fervenza FC, Ytterberg SR, et al. Diffuse alveolar hemorrhage secondary to antineutrophil cytoplasmic antibody-associated vasculitis: predictors of respiratory failure and clinical outcomes. Arthritis Rheumatol. 2016;68(6):1467-76.

55. Mohammad AJ, Mortensen KH, Babar J, Smith R, Jones RB, Nakagomi D, et al. Pulmonary involvement in antineutrophil cytoplasmic antibodies (ANCA)-associated vasculitis: the influence of ANCA subtype. J Rheumatol. 2017;44(10):1458-67. This study investigated pulmonary involvement in 140 patients with ANCA-associated vasculitis and found different patterns of abnormalities exclusively in patients with PR3-ANCA and MPOANCA vasculitis.

56. Nada AK, Torres VE, Ryu JH, Lie JT, Holley KE. Pulmonary fibrosis as an unusual clinical manifestation of a pulmonary-renal vasculitis in elderly patients. Mayo Clin Proc. 1990;65(6):847-56.

57. Arimura Y, Minoshima S, Tanaka U, Fujii A, Kobayashi M, Nakabayashi K, et al. Pulmonary involvement in patients with myeloperoxidase specific-antineutrophil cytoplasmic antibody. Ryumachi. 1995;35(1):46-55.

58. Nozu T, Kondo M, Suzuki K, Tamaoki J, Nagai A. A comparison of the clinical features of ANCA-positive and ANCA-negative idiopathic pulmonary fibrosis patients. Respiration. 2009;77(4):40715. 
59. Comarmond C, Crestani B, Tazi A, Hervier B, Adam-Marchand S, Nunes $\mathrm{H}$, et al. Pulmonary fibrosis in antineutrophil cytoplasmic antibodies (ANCA)-associated vasculitis: a series of 49 patients and review of the literature. Medicine (Baltimore). 2014;93(24):340-9.

60. Schirmer JH, Wright MN, Vonthein R, Herrmann K, Nölle B, Both $\mathrm{M}$, et al. Clinical presentation and long-term outcome of 144 patients with microscopic polyangiitis in a monocentric German cohort. Rheumatology (Oxford). 2016;55(1):71-9.

61. Eschun GM, Mink SN, Sharma S. Pulmonary interstitial fibrosis as a presenting manifestation in perinuclear antineutrophilic cytoplasmic antibody microscopic polyangiitis. Chest. 2003;123(1):297301.

62. Ando M, Miyazaki E, Ishii T, Mukai Y, Yamasue M, Fujisaki H, et al. Incidence of myeloperoxidase anti-neutrophil cytoplasmic antibody positivity and microscopic polyangitis in the course of idiopathic pulmonary fibrosis. Respir Med. 2013;107(4):608-15.

63. Alba MA, Flores-Suárez LF, Henderson AG, Xiao H, Hu P, Nachman PH, et al. Interstital lung disease in ANCA vasculitis. Autoimmun Rev. 2017;16(7):722-9.

64. Sebastiani M, Manfredi A, Vacchi C, Cassone G, Faverio P, Cavazza A, et al. Epidemiology and management of interstitial lung disease in ANCA-associated vasculitis. Clin Exp Rheumatol. 2020;38 Suppl 124(2):221-31.

65.• McDermott G, Fu X, Stone JH, Wallwork R, Zhang Y, Choi HK, et al. Association of cigarette smoking with antineutrophil cytoplasmic antibody-associated vasculitis. JAMA Intern Med. 2020;180(6):870-6. A dose-response relationship of cigarette smoking and ANCA-associated vasculitis risk was reported. This association was particularly strong among patients with MPO-ANCA vasculitis (former and current smokers).

66. Distler O, Highland KB, Gahlemann M, Azuma A, Fischer A, Mayes MD, et al. Nintedanib for systemic sclerosis-associated interstitial lung disease. N Engl J Med. 2019;380(26):2518-28.

67. Alba MA, Jennette JC, Falk RJ. Pathogenesis of ANCA-associated pulmonary vasculitis. Semin Respir Crit Care Med. 2018;39(4): 413-24.

68. Mahr A, Katsahian S, Varet H, Guillevin L, Hagen EC, Höglund P, et al. Revisiting the classification of clinical phenotypes of antineutrophil cytoplasmic antibody-associated vasculitis: a cluster analysis. Ann Rheum Dis. 2013;72(6):1003-10.

69. Unizony S, Villarreal M, Miloslavsky EM, Lu N, Merkel PA, Spiera R, et al. Clinical outcomes of treatment of anti-neutrophil cytoplasmic antibody (ANCA)-associated vasculitis based on ANCA type. Ann Rheum Dis. 2016;75(6):1166-9.

70. Geetha D, Jin Q, Scott J, Hruskova Z, Hanouneh M, Little MA, et al. Comparisons of guidelines and recommendations on managing antineutrophil cytoplasmic antibody-associated vasculitis. Kidney Int Rep. 2018;3(5):1039-49.

71. Charles P, Perrodeau É, Samson M, Bonnotte B, Néel A, Agard C, et al. Long-term rituximab use to maintain remission of antineutrophil cytoplasmic antibody-associated vasculitis: a randomized trial. Ann Intern Med. 2020;173(3):179-87. This randomized controlled trial found that patients who are in remission after 28 months benefit from additional rituximab infusions (relapse-free survival at 28 months of $96 \%$ compared to $74 \%$ in the placebo arm). In the placebo group, relapse was more common in PR3-ANCA vasculitis (10/25 patients versus 2/17 patients with MPO-ANCA vasculitis).

72. Jones RB, Hiemstra TF, Ballarin J, Blockmans DE, Brogan P, Bruchfeld A, et al. Mycophenolate mofetil versus cyclophosphamide for remission induction in ANCA-associated vasculitis: a randomised, non-inferiority trial. Ann Rheum Dis. 2019;78(3): 399-405.

73.•- Jayne DRW, Bruchfeld AN, Harper L, Schaier M, Venning MC, Hamilton $\mathrm{P}$, et al. Randomized trial of $\mathrm{C} 5 \mathrm{a}$ receptor inhibitor avacopan in ANCA-associated vasculitis. J Am Soc Nephrol.
2017;28(9):2756-67. In this phase 2 trial, the addition of avacopan to an induction regimen of rituximab or cyclophosphamide + low-dose prednisone or without prednisone was non-inferior to a standard of care treatment regimen. Adverse events were similar among the three groups of patients.

74. Kronbichler A, Leierer J, Gauckler P, Shin JI. Comorbidities in ANCA-associated vasculitis. Rheumatology (Oxford). 2020;59(Supplement_3):iii79-83.

75. Morgan MD, Turnbull J, Selamet U, Kaur-Hayer M, Nightingale P, Ferro CJ, et al. Increased incidence of cardiovascular events in patients with antineutrophil cytoplasmic antibody-associated vasculitides: a matched-pair cohort study. Arthritis Rheum. 2009;60(11): 3493-500.

76.• Wallace ZS, Fu X, Harkness T, Stone JH, Zhang Y, Choi H. Allcause and cause-specific mortality in ANCA-associated vasculitis: overall and according to ANCA type. Rheumatology (Oxford). 2020;59(9):2308-15. https://doi.org/10.1093/rheumatology/ kez589. This retrospective cohort study found that in comparison to patients with PR3-ANCA vasculitis, MPOANCA positive patients had a higher risk of cardiovascular disease-related death, even after adjustment for confounders.

77. Wallace ZS, Fu X, Liao K, Kallenberg CGM, Langford CA, Merkel PA, et al. Disease activity, antineutrophil cytoplasmic antibody type, and lipid levels in antineutrophil cytoplasmic antibodyassociated vasculitis. Arthritis Rheumatol. 2019;71(11):1879-87.

78. Bramlage CP, Kröplin J, Wallbach M, Minguet J, Smith KH, Lüders S, et al. Management of cardiovascular risk factors in patients with ANCA-associated vasculitis. J Eval Clin Pract. 2017;23(4):747-54.

79. Merkel PA, Lo GH, Holbrook JT, Tibbs AK, Allen NB, Davis JC, et al. Brief communication: high incidence of venous thrombotic events among patients with Wegener granulomatosis: the Wegener's Clinical Occurrence of Thrombosis (WeCLOT) study. Ann Intern Med. 2005;142(8):620-6.

80. Allenbach Y, Seror R, Pagnoux C, Teixeira L, Guilpain P, Guillevin L, et al. High frequency of venous thromboembolic events in Churg-Strauss syndrome, Wegener's granulomatosis and microscopic polyangiitis but not polyarteritis nodosa: a systematic retrospective study on 1130 patients. Ann Rheum Dis. 2009;68(4):564-7.

81. Stassen PM, Derks RP, Kallenberg CG, Stegeman CA. Venous thromboembolism in ANCA-associated vasculitis-incidence and risk factors. Rheumatology (Oxford). 2008;47(4):530-4.

82. Novikov P, Makarov E, Moiseev S, Meshkov A, Strizhakov L. Venous thromboembolic events in systemic vasculitis. Ann Rheum Dis. 2015;74(3):e27.

83. Kronbichler A, Leierer J, Leierer G, Mayer G, Casian A, Höglund $\mathrm{P}$, et al. Clinical associations with venous thromboembolism in antineutrophil cytoplasm antibody-associated vasculitides. Rheumatology (Oxford). 2017;56(5):704-8.

84. Kang A, Antonelou M, Wong NL, Tanna A, Arulkumaran N, Tam FWK, et al. High incidence of arterial and venous thrombosis in antineutrophil cytoplasmic antibody-associated vasculitis. J Rheumatol. 2019;46(3):285-93.

85. Berti A, Matteson EL, Crowson CS, Specks U, Cornec D. Risk of cardiovascular disease and venous thromboembolism among patients with incident ANCA-associated vasculitis: a 20-year population-based cohort study. Mayo Clin Proc. 2018;93(5):597-606.

86. Kronbichler A, Leierer J, Shin JI, Merkel PA, Spiera R, Seo P, et al. Association of pulmonary hemorrhage, positive proteinase 3, and urinary red blood cell casts with venous thromboembolism in antineutrophil cytoplasmic antibody-associated vasculitis. Arthritis Rheumatol. 2019;71(11):1888-93. In this sub-analysis of the RAVE trial, PR3-ANCA positivity, pulmonary hemorrhage, and the presence of red blood cell casts in the urine 
associated with an increased risk of venous thromboembolic events in patients with ANCA-associated vasculitis.

87. Henry N, Brilland B, Wacrenier S, Djema A, Garnier AS, Gansey $\mathrm{R}$, et al. Incidence and risk factors of venous thromboembolic events in patients with ANCA-glomerulonephritis: a cohort study from the Maine-Anjou Registry. J Clin Med. 2020;9(10).

88. Bautz DJ, Preston GA, Lionaki S, Hewins P, Wolberg AS, Yang JJ, et al. Antibodies with dual reactivity to plasminogen and complementary PR3 in PR3-ANCA vasculitis. J Am Soc Nephrol. 2008;19(12):2421-9.

89. Hilhorst M, Winckers K, Wilde B, van Oerle R, ten Cate H, Tervaert JW. Patients with antineutrophil cytoplasmic antibodies associated vasculitis in remission are hypercoagulable. $\mathrm{J}$ Rheumatol. 2013;40(12):2042-6.

90. Orsi FA, Cannegieter SC, Lijfering WM. Statin therapy to revert hypercoagulability and prevent venous thromboembolism: a narrative review. Semin Thromb Hemost. 2019;45(8):825-33.

91. Craven A, Robson J, Ponte C, Grayson PC, Suppiah R, Judge A, et al. ACR/EULAR-endorsed study to develop Diagnostic and Classification Criteria for Vasculitis (DCVAS). Clin Exp Nephrol. 2013;17(5):619-21.

Publisher's Note Springer Nature remains neutral with regard to jurisdictional claims in published maps and institutional affiliations. 